\title{
Great Deluge and Extended Great Deluge based job scheduling in grid computing using GridSim
}

\begin{abstract}
Scheduling of jobs is one of the most important research areas of Grid computing as it has attracted so much attention since its beginning. Job scheduling in Grid computing is a NPComplete problem due to Grid characteristics such as heterogeneity and dynamicity. Many heuristic algorithms have been proposed for Grid scheduling to avail Grid computing. However, these heuristic methods are limited by time constraints required for remapping of jobs to Grid resources in such elastic and dynamic environments. Great Deluge (GD) is a practical solution for such a problem. Therefore, this paper presents Great Deluge and Extended Great Deluge (EGD) based scheduling algorithm for Grid computing. We also present the detailed implementation of GD and EGD in a reliable simulation platform, GridSim. This has two advantages. First, it will ease the reimplementation process for future contributors since there are lots of complexity and ambiguity to develop such scheduling algorithms. Second, most of the research and experimental results, especially in the area of Grid scheduling, have used their own developed infrastructure to simulate the performance of their algorithms, thus the question remains on how well they will perform in a real world environment. We also, investigate the computation time and the number of soft constraints violations of EGD against its conventional GD algorithm. The GD scheduling algorithm is able to provide qualitative solution in shorter time for small Grid size while EGD could produce schedule in shorter time for all cases.
\end{abstract}

Keyword: Grid scheduling; Great Deluge; Heuristics; GridSim 